\title{
5 Our Quantized Reality: Life in the Strobe Lights
}

Although we perceive that, generally, everyday life and things are continuous - energy, movement, etc., a bit of scientific analysis of numerous phenomena shows that in many cases it just cannot possibly be so. This realization gave rise a few decades ago to the new science of quantum mechanics. Quantum mechanics has proved so far to be hands down the most successful theory in the history of physics in explaining reality, but it's also by far the weirdest, leading many to question whether this can be the ultimate explanation of reality.

Historically, quantum mechanics arose to try and explain how atoms and electrons behave. Matter is made up of atoms, i.e. electrons spinning around nuclei. But how can electrically charged electrons go on spinning, at high speed, without steadily losing energy? According to all the laws of classical physics, electrons ought to slowly wind down as their motion should lead to a never-ceasing leakage of energy. Straightforward calculations in classical physics tell us that they would quickly collapse onto the nucleus - which they obviously do not.

Neither human intuition nor classical mechanics can describe nor explain how matter behaves at that level, but quantum mechanics does (25).

In the illustrative case of the atom, quantum physics demonstrates that there are a few privileged individual orbits around the nucleus within which electrons never lose their energy, regardless of speed. (As mentioned earlier, the modern description of electrons is a bit more complex, as electrons are described as quantum excitations within a field rather than physical particles. This modern description does not invalidate this discussion.).

But it also proves that any other orbits other than those few privileged spinning orbits are no-go zones for the electrons; indeed, electrons can never be found at any such other orbits. Different 'allowed' orbit levels around the nucleus correspond to different energy levels of the spinning electron: The further away from the nucleus an electron spins, the higher its energy level.

This property is by now well-known and is routinely harnessed in mundane applications such as light bulbs; when electricity is fed into a bulb filament its energy is used to move electrons up to higher orbits. Electrons on these higher orbits are perched precariously on too-high orbits and tend to 'fall back' to a more comfortable, less excited lower orbit. To do so, however, they need to shed energy which they do in the form of light - presto, an illuminated light bulb.

Beside the few allowed orbits where electrons may be present, the rest of space is taken up by no-go spatial zones above and beneath every allowed level. If electrons somehow could ever stray into the unallowed zones, they would be instantly destroyed - as would then matter itself.)

Whenever an electron jumps to a higher level (because it absorbed energy from the electrical power fed into the light bulb) and when it then jumps back down to a lower orbit (releasing energy in the form of light) it does not, and cannot, actually jump: it seemingly dematerializes from one level and rematerializes in the new orbit. This seems to be nonsense, of course, and it is - all the attempted physical explanations of this phenomenon break down at one level 
or another, unless the explanation is taken to be purely mathematical and that electrons are viewed, as they now are, as purely non-physical excitation states of a mathematical field (26). It can be said, therefore, that electrons are not objects at all, but mathematical entities, quantum 'excitations' of an all-pervading field, the fermionic matter field. A field is a purely mathematical construct - a mathematical description of reality (27).

The ultimate explanation for all known physical phenomena appears to be ultimately purely mathematical. Both straightforward as well as sometimes seemingly weird properties of the physical world arise just so, because of nature's obedience to mathematics.

Let's illustrate briefly just how pervasive the presence of quanta and of quantization - lack of smooth continuity - is in our everyday lives and how surprising and often counterintuitive it can be. Consider something very simple and commonplace, somewhat cumbersomely called the quantity of movement.

The 'quantity of movement' (QoM) of an object is defined as the product, i.e. the result of the multiplication, of the object's mass by its speed: the instantaneous quantity of movement of, say, your arm at any point in time is thus the product of its mass by its instantaneous speed. When your arm is at rest, its speed is zero, and therefore its quantity of movement at this particular moment in time is zero: its mass (broadly, its weight) times its speed (zero). Since its speed is zero, then its QoM is the product of zero times something (its mass), hence it is also equal to zero.

Now move your arm from a first position at rest (where its speed is zero) to another position at rest (where its speed becomes again zero). Its quantity of movement started from naught, rose up, went through a peak before decreasing and finally coming back to zero again when the arm comes again to rest. If the value of its quantity of movement were plotted against time, the graph would appear more or less bell-shaped.

Nothing special there except that the values of its quantity of movement are not continuous at all, but are discontinuous: the QoM kept 'leaping' in tiny discrete jumps from an authorized value to another authorized value. Of course, the individual jumps are extremely tiny and the 'authorized' values are numerically very close thus lending the illusion, at a macroscopic level, of smoothly continuous quantity of movement values.

The words ‘quantized' and 'discrete' are sometimes used differently, although both words mean discontinuous. All dynamic processes in the world are quantized (such as the quantity of your arm's movement). This does not mean however that the underlying environment - the spacetime - within which your arm moves is necessarily itself quantized: the weft of the space-time within which your arm moves could well be continuous, or not. The quantization of a dynamic event or process is more often referred to as its 'quantizedness' or quantization, whereas if the weft or fabric of the 'background stage' itself, such as the space or time within which some events happens, is itself discontinuous, this granular quality of the background is more usually designated by 'discreteness'. 'Discrete' is also sometimes called 'granular'. 'Granularity' often refers to the dimensions or size of the discrete parameters, whereas 'discreteness' refers to the quality of being discontinuous, irrespective of the associated values. 
Why are so many things discrete in nature? There is a simple answer that the quantization of many things in nature comes from the presence of finite boundaries encasing the sweep or scope within which these things can exist and evolve in time. To understand how it works, consider a rope.

Let's fasten the end of a rope to a wall, take the other end and shake it up and down. A pattern of stationary waves will quickly form with the rope taking on the broad shape of a stationary sinusoidal curve (a sine curve). If we lazily shake up and down the loose end of the rope, it will take on the shape of a half sinusoidal curve, with only one point at rest (the rope end fastened to the wall). Now if we shake the unfastened end more forcefully (i.e. input more energy to the rope) a pattern will form whereby a new stationary, immobile point will form in the middle of the rope rather than only at the wall end. This comes about because 'amplitude waves' travel to the wall from your hand and reflect back, and the forward and backward waves interfere with each other.

The amplitudes travelling back and forth cancel out at some point on the rope and create an immobile point. The more energetically we shake our end of the rope, the more immobile points form along the rope. The rope takes on a stationary sinusoidal shape, with more points and higher energy travelling back and forth along it. Note that the formation of a sinusoidal pattern along the rope does not even depend on the end point being fixed: one could dangle the rope in a void and shake it, say from a high vantage point, and a similar pattern would still form - with the far end of the rope taking on the position of maximum, rather than zero, amplitude.

This pattern formation arises solely because the rope is finite in length, not because its end point is fixed, and the waves that you send travelling down the rope by shaking it reflect back off the rope end regardless of whether that end is fixed or loose.

By shaking one end of the finite-length rope, what we have created is a quantization of the rope's movement amplitudes (and several other attributes besides). Only certain energies can stably create this quantization (extra energy might be reflected back to you and travel up your arm) and the quantization happens in the first place only because the existence of the rope's boundaries enable quantization.

This principle is general: quantization of something's attributes or its properties is often created by finiteness, and continuums blossom best within infinite environments (the precise conditions of applicability of this broad principle depend on the particular attributes at hand. There might exist continuums of certain parameters or properties within our finite universe).

Another telling and equivalent way to look at the role of finiteness in creating quantization is to look at numbers: Unless we extend any number to infinity, there is no continuity in numbers and they proceed from one number to the next number in line by quantum jumps: If we keep, say, 30 decimal places after a comma, then the number, say

\subsection{1}

moves on to the next one in line, to wit 
thus by a ‘quantum jump’ of size:

0.000000000000000000000000000001.

Only by accepting an infinite number of decimal places after a comma can we render the set of all numbers seamlessly continuous. Again, only infinity (thanks to its absence of boundaries) removes quantization, i.e. the discontinuous jumps from one value to the next in line.

One of the possible explanations we saw earlier to solve the conundrum of the present was quantized time. Under this scenario the present would exist and simply be the quantum of time, with time moving in quantum jumps from one now to the next. Some of the current approaches in the field of quantum gravity (28) precisely lead to the view that space-time, and individually space and time, are discrete.

Let's now consider other ways whereby reality can be rather odd, even if we often fail to realize it, so used are we to the way the world works that we take it unquestioningly in our stride. Armed with a new awareness of how odd reality can be, we'll then seek an explanation for reality, and find a possible one. 